\title{
The case for matching MHC genes in human organ transplantation
}

\author{
Susan Martin \& Philip A. Dyer
}

NW Regional

Tissue Typing Laboratory, St. Mary's Hospital, Manchester M13 OJH, UK

\begin{abstract}
Following Medawar's demonstration of the genetic basis to organ transplant rejection, early pioneers of human clinical organ transplantation attempted to ensure success either by transplanting between genetically identical individuals or by suppressing the vigorous host immune response. Genetic identity was ensured through monozygotic twin live donor kidney transplantation as pioneered in Boston, in the 1950s. The inevitable aggressive immune response in genetically mismatched cadaver donor kidney and liver transplantation was suppressed by use of steroids and azathioprine. In the 1960 s, when the highly polymorphic human major histocompatibility system (Mhc) genes (HLA alleles) and their corresponding cell surface antigens (specificities) were dis-
\end{abstract}

covered, it became possible to use siblings who were 2 or 1 haplotype matches as living kidney donors, thus enlarging the donor pool.

The early days of matching for HLA specificities stimulated a controversy of efficacy which remains today ${ }^{1-4}$. The main reason for the debate is the inability to perform a controlled trial of HLA matched versus non-matched transplants without the confusing interference of prophylactic immunosuppression. All organ transplant recipients (except very rare monozygotic twin kidney transplants) receive at least one, and usually several, of an increasing array of expensive drugs all of which powerfully suppress the recipient's ability to mount an effective immune response acting at various levels from inhibiting RNA synthesis to restricting
$\mathrm{T}$ and $\mathrm{B}$ cell functions.

HLA antibodies and rejection It is quite clear that transplantation of a kidney which expresses an HLA specificity into a patient with preformed antibodies directed against that specificity leads to immediate failure by hyperacute rejection. This is also true, but perhaps to a lesser degree in liver and heart transplants which are, unlike renal transplantation, essentially one-off procedures. This central role of HLA specificities in kidney transplantation has not been questioned and since the mid 1960s a pre-transplant crossmatch between donor and recipient has always been performed to detect and prevent early, rapid transplant failure. Some kidney, heart and liver transplant programmes have

\section{Is MHC matching as a primary criterion in kidney allocation justified?}

\author{
Arthur J. Matas
}

Department of Surgery, University of Minnesota, Minneapolis, Minnesota 55455, USA
There are numerous goals to be satisfied when compiling a list of criteria for the choice of kidneys in transplantoperations: to demonstrate objectivity, to improve transplant outcome, to be fair to all on the waiting list, to be timely (kidneys have a finite preservation time) and to limit costs. These goals may conflict. For example, a system that maximizes outcome may be unfair or extremely costly. In the United States, the United Network for Organ Sharing (UNOS) has developed a point system for cadaver kidney allocation. General rules specify the use of blood group $O$ kidneys for type $\mathrm{O}$ recipients and the national sharing of 6-antigenmatched or phenotypically identical kidneys. Non identical kidneys are used locally; points are assigned for histocompatibility matching, sensitization, and waiting time. In parts of
Europe, histocompatibility matching is the major criterion for allocation and a proposal for a similar system has been made in the United States ${ }^{1}$. Does allocation based on histocompatibility alone come closer to satisfying the five goals as outlined above?

\section{Objectivity}

The process should be seen to be without bias. Observers should know exactly why any one kidney was allocated to any one patient. Allocation by histocompatibility matching is theoretically objective: every individual has defined HLA -A, $-B$ and -DR antigens and potential recipients could be ranked by the number of donor-recipient matches. However, an argument for allocation based on the objectivity of matching implies that the determination of donor and recipient HLA-A, - B and $\mathrm{DR}$ antigens is always accurate. The Collaborative Transplant Study Group recently compared serologic and DNA typing for 3,325 donors and 4,076 recipients: serologic typing for DR was inaccurate for $25 \%$ of organ donors and $27.6 \%$ of recipients ${ }^{2}$. Individual centres had discrepancy rates between $9.7 \%$ and $86.7 \%$. Thus, the overall chance of an error in donor and/or recipient typing is about $46 \%$. With such a high rate of error, it is unreasonable to use matching as a major criterion for allocation.

\section{Transplant outcome}

Data on the impact of matching on transplant outcome are controversial. Proponents of a major role for matching argue that well-matched grafts do better because there is a 
Table 1 Effect of HLA-DR antigen matching on transplant survival

\begin{tabular}{|c|c|c|c|c|}
\hline \multirow{2}{*}{$\begin{array}{c}\text { HLA-DR } \\
\text { Mismatches }\end{array}$} & \multirow{2}{*}{$\begin{array}{l}\text { Number } \\
\text { of cases }\end{array}$} & \multicolumn{3}{|c|}{$\%$ Acturial transplant survival $^{15}$} \\
\hline & & 1 year & 5 years & 10 years \\
\hline Zero & 422 & 85.4 & 72.1 & 66.1 \\
\hline One & 438 & 79.8 & 64.0 & 51.8 \\
\hline Two & 140 & 67.8 & 52.8 & 40.4 \\
\hline
\end{tabular}

also shown that when transplants are mismatched, recipients produce antibodies directed to the mismatched HLA specificity and even with sophisticated immunosuppressive drug therapy such antibody production is associated with transplant failure ${ }^{5,6}$. When repeat transplantation is needed such antibody formation seriously reduces the chance of finding a suitable donor.

Centres advocating allocation of organs to recipients on the basis of least HLA mismatch cite studies showing that transplant survival is significantly improved in cases where a high degree of HLA matching is achieved over cases where there

better outcome in living related donor (versus cadaver) transplants and outcome is better in well-matched (versus poorly matched) cadaver transplants. These arguments need detailed analysis. Although living related donor transplants do have better outcomes than cadaver transplants, the reason may not be better histocompatibility matching. In fact, other than the perfectly matched living related donor ( 2 antigens matched at each of HLA-A, $-B$ and -DR), no evidence exists that matching has any impact on the outcome of living donor transplants; 1 -haplotype ( 3 antigens) and 0 haplotype ( 0 antigens) matched living related donor transplants do equally well ${ }^{3}$. More importantly, living unrelated donor transplants - which are no better histocompatibity matched than cadaver transplants have outcomes similar to living related donor transplants ${ }^{4,5}$. With a living donor (unrelated or related) initial graft function is excellent and patient care much easier. It may be that this early function is the cause of the better outcome. The prognosis is similarly is little or no HLA match ${ }^{7}$. In our own centre (see Table 1) where allocation of cadaver kidneys has always been based on HLA matching, we find that those which had no mismatches for HLA-DR specificities have a transplant survival of $85.4 \%$ after one year. This is $17.6 \%$ higher than complete (two) HLA-DR mismatched transplants. The remaining cases with one HLA-DR mismatch show

\begin{tabular}{l} 
Allocating organs \\
No one disputes that the degree with which \\
a donated organ matches the HLA status of \\
the recipient is relevant to the well being of \\
both the donated organ and the patient. \\
However, it is a mystery how different \\
countries can have evolved such divergent \\
HLA matching practices. By way of \\
advancing the discussion, two contrasting \\
views on the practice of HLA matching are \\
presented (pages 210 to 213 ). Matas makes \\
the case for only a limited recognition of the \\
HLA match between donor and recipient \\
(broadly the practice in the U.S.), whereas \\
Martin and Dyer (representing a European \\
point of view) argue that HLA matching is the \\
most important factor to be considered.A.J.I. \\
\hline
\end{tabular}

good in the subgroup of cadaver kidneys with excellent initial function (irrespective of matching) . $^{6}$

In the United States, the impact of matching on cadaver transplant outcome is small (Fig. 1). It has been shown clearly that perfectly matched (6-antigen-match) trans-plants have better outcome ${ }^{1}$, and current policy mandates the national sharing of such kidneys. But there is little evidence to support giving priority to histocompatibility matching for other kidneys. In a multifactorial analysis of 35,625 kidney transplants reported to the UNOS transplant registry between 1988 and 1991 (ref. 7), the dominant factor influencing outcome was the centre where the transplant was done. Matching did affect outcome but the impact was small: at intermediate survival. Highly significant improvements in graft survival (up to 10 years post transplantation) have also been observed for HLA-DR matched donor-recipient pairs (Fig. 1).

Matching - a valuable resource Arguments against selection of organ recipients based on HLA matching protocols centre on the difficulty of achieving a match due to the highly polymorphic nature of the $\mathrm{MHC}$; over 150 alleles of HLA-A, -B, -C and -DR genes exist and HLA alleles can be population specific, or at least of unequal distribution between different ethnic groups. This has precipitated considerable debate in the United States where most cadaver organ donors are of caucasoid origin. It has to be remembered that the number of cadaveric organs available for transplantation has stabilized, world wide, at approximately 20 per million population per year. Organ donation at this rate will never meet recipient demand. In such a situation, it is essential to maximise the efficiency of

3 months post-transplant, graft survival for organs with 1 mismatch at HLA-A or - B was $88.5 \%$; 2 mismatches, $87.1 \%$; 3 mismatches, $86.1 \%$; and 4 mismatches, $85 \%$. 3-month survival for 1 HLA-DR mismatch was $86.9 \%$ and for 2 DR mismatches was $85.7 \%$. Because of the large numbers in each subgroup, these differences - though minor - are statistically significant. The subsequent impact of matching was also studied. For recipients whose grafts functioned at 3 months, 12month survival for 1 mismatch at HLA-A or -B was $94.2 \%$; for 2 mismatches, $92.9 \%$; for 3 mismatches, $92.1 \%$; and for 4 mismatches, $92 \%$. DR matching was not found to be a long-term factor in graft survival ${ }^{8-10}$. In a review of the United States Renal Data System $(17,913$ cadaver and 7,061 living donor transplants), the maximum possible impact of an allocation system based on matching was a $3 \%$ change in 5-year graft survival $^{10}$. Our data $(1,329$ cadaver transplants) show that HLA matching has no impact on short-term graft survival, long-term graft survival or 


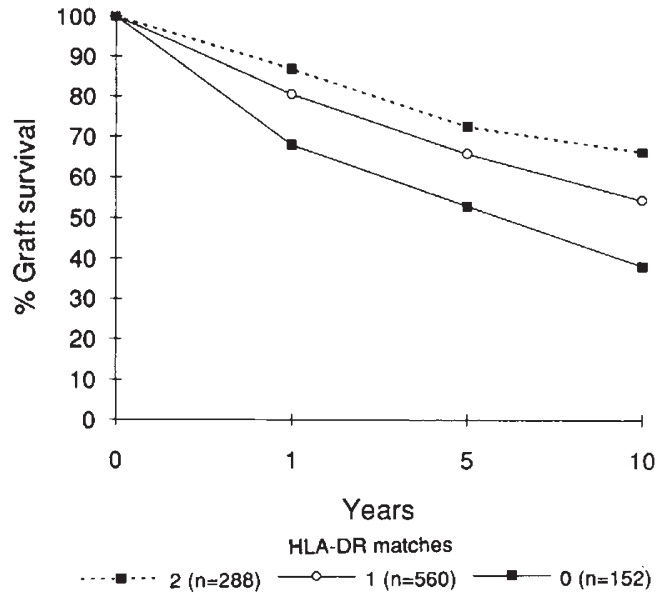

Fig. 1 The effect of HLA-DR matching donors and recipients on graft survival.

the rare resource available. Matching for HLA alleles and specificities cannot do anything but contribute positively toward that goal. Individuals with rare phenotypes may have to wait longer for a suitable matched donor but limited, effective matching, perhaps just for HLA-DR specificities, is possible given that the HLA-DR polymorphism is less than that for HLA-A and HLA-B or all three loci combined.

Some single centres have failed to find a benefit from matching for $\mathrm{HLA}^{8}$, this is to be expected occasionally given the powerful effect of immunosuppressive therapy, but it cannot be used as evidence to advocate a universal mismatching policy. An important factor between single centre experiences is the overall survival rates achieved, which can vary from $75-95 \%$ at one year for kidney transplants. Furthermore, studies of HLA matching effects vary between centres which achieve a high degree of deliberate pre-transplant matching and those with random allocation and consequent HLA mismatching.

\section{Molecular typing techniques}

The techniques available to identify HLA specificities were standardised on serological methods. These are now being superceded following recent advances in molecular biology. Highly specific and rapid methods, such as poly- $a$

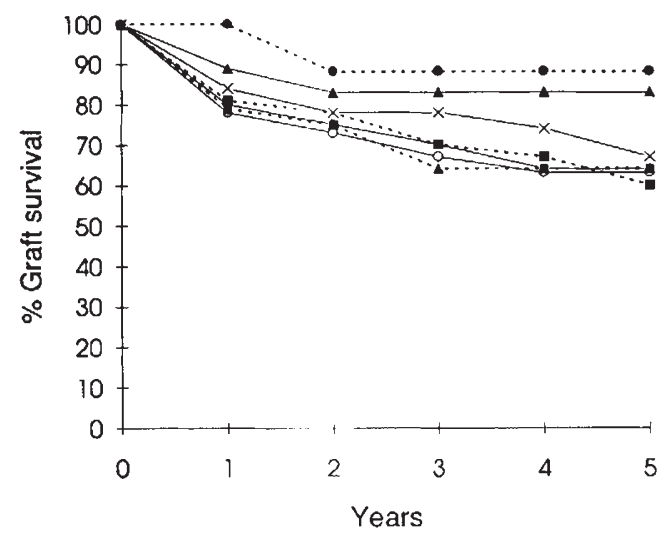

$b$
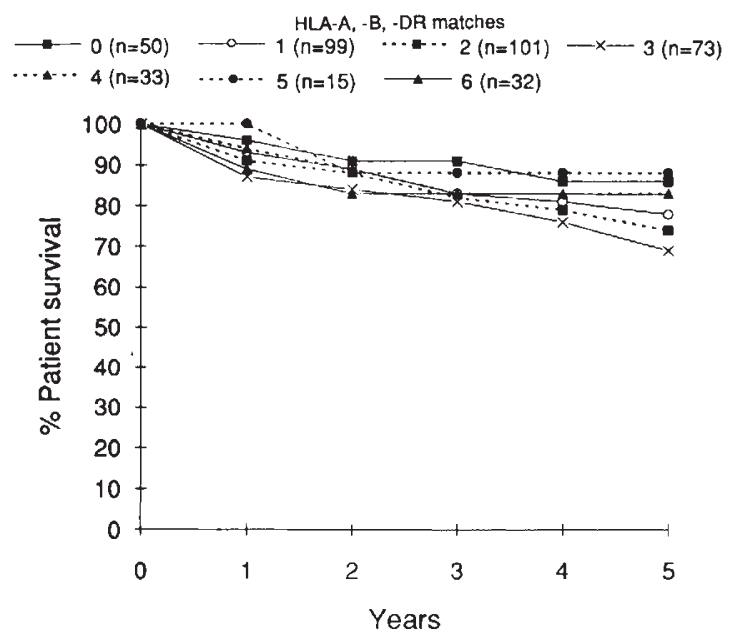

development of antibodies to HLA antigens after graft failure ${ }^{11}$.

\section{Impartiality}

A system based solely on HLA matching is unfair to those patients with rare HLA types. For

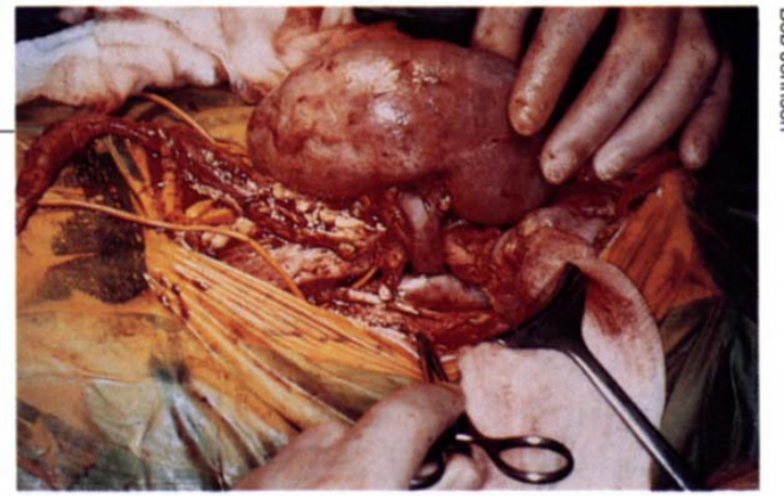

Immediately following engraftment the kidney is engorged and has a healthy pinkish appearance.

example, frequencies of HLA antigens differ in African-Americans and Caucasians. In the United

Fig. 1 a, Actuarial graft survival for first cadaver transplant recipients at the University of Minnesota since 1988 and subgrouped by HLA-ABDR match. There is a significant difference between the 0 -antigen versus 6 -antigen-matches and the 1 antigen versus 6 -antigen-matches $(p<.05)$. There is a trend to better outcome for the 5-antigen-matches (NS, but small numbers). There are no other differences. $b$, Patient survival for first cadaver transplant recipients at the University of Minnesota since 1988 and subgrouped by HLA-ABDR match. There are no significant differences between groups. merase chain reaction amplification using sequence specific primers (PCR-SSP ${ }^{9}$ ) exist and have reduced errors in typing both patients and donors considerably. Already, retrospective studies of HLA matching in kidney transplantation using molecular biological techniques have revealed increased survival rates in transplants with no HLA- DR mismatches at the allele level ${ }^{10}$. In our own centre we have found that molecular and serological typing techniques give concordance rates in excess of $90 \%$ with the discordance being largely restricted to those specificities that are recognized as difficult to define using serological techniques (such as the HLA-DR52 group). This degree of concordance has also been reported in a European multicentre study ${ }^{11}$. Initially, concordance rates were claimed to be lower but many reported discrepancies were frequently errors in verbal transmission of data between transplant centres, quite unrelated to

\section{管}

States, the majority of donors are Caucasian $(>80 \%)$ and allocation based on matching alone would lead to prolonged waiting times for African-Americans.

\section{Preservation time}

Shipping kidneys to the bestmatched recipient will increase preservation time. There is a finite time in which kidneys can be preserved and still transplanted. Preserving kidneys for more than 20 hours is associated with decreased graft survival ${ }^{7}$. Most centres attempt to transplant kidneys within 48 hours of removal. Moreover, when kidneys are shipped to the best patient match without prior crossmatching (that 
techniques used and can be avoided simply by better communication. Thus, new and more accurate techniques enable more precise matching by defining individual alleles which influence transplant outcome. In the past and using serological assays, some centres have not achieved acceptable HLA typing standards. This must have influenced retrospective studies of $\mathrm{HLA}$ matching effects.

Traditionally, HLA matching has been applied to only kidney transplantation and in Europe matching is the standard practice. Several studies of clinical parameters of HLA mismatching in liver, heart, lung and pancreas transplants (such as incidence and degree of rejection, appearance of circulating antibodies to mismatched donor HLA specificities, short and long term survival and occurrence of posttransplant diseases) have shown correlations with donor-recipient HLA mismatching. Thus the overall

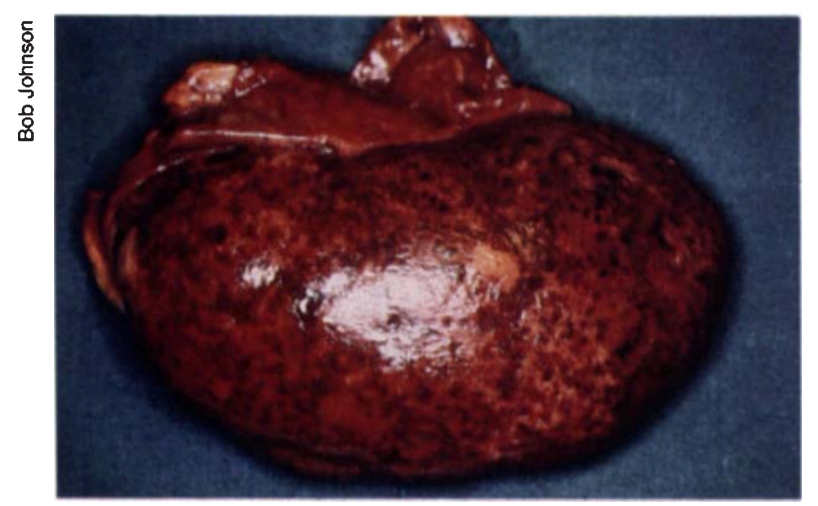

Within minutes of engraftment the acutely rejected kidney takes on a dramatic appearance

is, testing of recipient serum for preformed antibodies to the donor) the potential recipient may well have antibodies to the donor kidney. If so, someone other than the designated recipient must be found to receive the shipped kidney. Under these circumstances, time is wasted and the excercise may prove futile.

\section{Cost}

Allocation of kidneys based on matching increases transplantation costs, due to the expense of shipping, the increased time for preservation personnel and in some cases the necessity for repeat crossmatching. There is a moral cost also whenever effect of HLA mismatching is probably greater than often reported.

The cost of organ transplantation is an issue which health care economists and the public are keen to monitor. It has been reported that costs at 1992 levels are $\$ 38,000$ for kidney, $\$ 89,000$ for heart and $\$ 132,000$ for liver transplantation ${ }^{12}$ while the laboratory cost for HLA matching is probably no more than $\$ 1500$. Thus, although any benefit to be gained from HLA matching is at very small additional cost to the overall expenditure, it can add $10 \%$ to survival rates and is therefore very cost effective. Informed members of the public would probably ask why such a cost effective procedure was not employed especially given that costs of re-transplantation, following a failed graft, are considerably higher.

With many transplant recipients surviving for more than 20 years the legacy of long-term immunosuppressive therapy can now be assessed. Sadly, this legacy is proving to be high rates of malignancies, in many cases ${ }^{13,14}$. These cancers are often aggressive and unusual in site, proving a challenge to treat. If HLA matching removes, or even reduces in any small way, the need for agressive and comprehensive long term immunosuppressive drug therapy, then not only will substantial savings be made but significant benefit can be obtained in ways that will affect both quality and longevity of transplanted organ function.

\section{References}

1. Braun, W.E. New Engl. J. Med. 327 883-885 (1992).

2. Matas, A.J. et al. Transplantation $50599-607$ (1990).

3. Opelz, G., Wujciak, T., Mytilineos, J. \& Scherer, S. Transplant. Proc. 25(1) 173-175 (1993).

4. Takemoto, S., Terasaki, P.I., Cecka, J.M., Cho, Y.W. \& Gjertson, D.W. New Engl. J. Med. 327 834-839 (1992)..

5. Martin, S. et al. Transplant. Proc. 29(1) 899-900 (1987).

6. Smith, J.D., Danskine, A.J., Laylor, R.M., Rose, M.L. \& Yacoub, M.H. Transplant. Immunol. 1 60-65 (1993).

7. Martin. S., Connolly, J., Parrott, N.R., Johnson, R.W.G. \& Dyer, P.A. Transplant. Proc. (in the press).

8. Opelz, G. Transplantation $53694-696$ (1992).

. Olerup, O. \& Zetterquist, H. Tissue Antigens 39 225- 235 (1992).

10. Poli, F. et al. Transplantation 56 97-100 (1993).

11. Verduyn, W. et al. Hum. Immunol. 37 59-67 (1993).

12. Evans, R.W., Manninen, Dong, F.B. \& McLynne, D.A. Transplant. Proc. 25(1) 1694-1696 (1993).

13. Cockfield, S.M., Preiksaitis, J.K., Jewell, L.D. \& Parfrey, N.A. Transplantation 56 88-96 (1993).

14. Sheil, A.G.R., Disney, A.P.S., Mathew, T.G., Amiss, N. \& Excell, L. Transplant. Proc. 24(5) 1946-1947 (1992).

15. Peto, R. et al. Br. J. Cancer 35 1-23 (1977). a local recipient (the patient who would have received the kidney if it was not shipped) is bypassed for the wrong reasons.

In animal models, matching significantly affects transplant outcome. But major differences exist between these models and the clinical situation. Animal experiments have used acute survival models with minimal long-term immunosuppression. In clinical practice all patients receive a combination of powerful immunosuppressive agents. In the presence of these agents when acute rejection is either averted or treated, immunoregulatory processes that enhance graft outcome may develop ${ }^{12,13}$. These processes may help explain why many poorly matched grafts do well.

All other parameters being equal, it cannot be bad to receive an HLAmatched allograft. Even though the observed impact of matching on outcome is small in the United States, an impact can nonetheless be seen. However, there are many other considerations to be taken into account. A system based on matching alone is not fair, practical or cost- effective, and, given the current inaccuracies in typing, is not objective. The marginal impact of matching for recipients with less than perfect matches does not justify its use as a primary criterion for cadaver kidney allocation.

\section{References}

1. Takemoto, S. et al. New Engl. J. Med. 327, 834839 (1992)

2. Mytilinos, J. et al. Transplantation 55, 778-781 (1993).

3. Kaufman, D.B., Sutherland, D.E.R., Noreen, H., Najarian, J.S. \& Fryd, D.S. Transplantation 47, 113-119 (1989).

4. Terasaki, P.I. et al. Clinical Transplants 1991 (eds Terasaki, P.I.\& Cecka, J.M.) 409-430 (UCLA Tissue Typing Laboratory, Los Angeles, 1992).

5. Park, K., Kim, Y.S., Lee, E.M., Lee, H.Y. \& Han, D.S. in Clinical Transplants 1992 (eds Terasaki, P.I. \& Cecka, J.M.) 249-256 (UCLA Tissue Typing Laboratory, Los Angeles, 1992).

6. Najarian, J.S. etal. Transplantation (in the press).

7. Gjertson, D.W. in Clinical Transplants 1992 (eds Gjertson, D.W. in Clinical Transplants 1992 (eds
Terasaki, P.I. \& Cecka, J.M.) 299-317 (UCLA Tissue Typing Laboratory, Los Angeles, 1993).

8. Gilks, W.R., Gore, S.M. \& Bradley, B.A. Transplantation 50, 141-146 (1990).

9. Thoroyood, J. et al. Transplantation 50, 146150 (1990).

10. Held, P.J. et al. American Society of Transplant Surgeons 16th Annual Meeting p91 (1991).

11. Matas, A.J. et al. Transplantation 50, 599-607 (1990).

12. Reinsmoen, N.L. \& Matas, A.J. Transplantation 55, 1017-1023 (1993).

13. Suciu-Foca, N. et al. Transplantation 51, 593 601 (1991). 